Published in final edited form as:

Prog Cardiovasc Dis. 2011 ; 54(3): 191-203. doi:10.1016/j.pcad.2011.09.004.

\title{
Assessment of Myocardial Ischemia with Cardiovascular Magnetic Resonance
}

\author{
Bobak Heydari, Michael Jerosch-Herold, and Raymond Y. Kwong ${ }^{\star}$ \\ Department of Cardiology, Brigham and Women's Hospital, Boston, MA 02215
}

\begin{abstract}
Assessment of myocardial ischemia in symptomatic patients remains a common and challenging clinical situation faced by physicians. Risk stratification by presence of ischemia provides important utility for both prognostic assessment and management. Unfortunately, current noninvasive modalities possess numerous limitations and have limited prognostic capacity. More recently, ischemia assessment by cardiovascular magnetic resonance (CMR) has been shown to be a safe, available, and potentially cost-effective alternative with both high diagnostic and prognostic accuracy. Cardiovascular magnetic resonance has numerous advantages over other noninvasive methods, including high temporal and spatial resolution, relatively few contraindications, and absence of ionizing radiation. Furthermore, studies assessing the clinical utility and cost effectiveness of CMR in the short-term setting for patients without evidence of an acute myocardial infarction have also demonstrated favorable results. This review will cover techniques of ischemia assessment with CMR by both stress-induced wall motion abnormalities as well as myocardial perfusion imaging. The diagnostic and prognostic performance studies will also be reviewed, and the use of CMR for ischemia assessment will be compared with other commonly used noninvasive modalities.
\end{abstract}

\section{Keywords}

Acute coronary syndrome; Adenosine; Cardiac magnetic resonance imaging (CMR); Chest pain; Coronary artery disease; Dobutamine; Echocardiography; Ischemia; Myocardial infarction; Perfusion; Persantine; Regadenoson; Positron emission tomography; Single-photon emission computed tomography; Vasodilators

The assessment of patients presenting with symptoms suspicious for myocardial ischemia remains one of the most common and challenging clinical scenarios faced by physicians. Over the past decades, there has been a significant reduction in the 30-day mortality for patients presenting with an acute coronary syndrome (ACS). ${ }^{1}$ Despite this considerable advancement in treatment, more than $50 \%$ of cardiac mortality from acute myocardial infarction (MI) occurs before reaching the cardiac catheterization laboratory, ${ }^{2}$ highlighting the important role of risk stratification in averting cardiac events. Although several clinical risk indices are available, imaging different aspects of ischemia by nuclear scintigraphy and echocardiography individually has been shown to have incremental but not powerful prognostic value. ${ }^{3-6}$

(C) 2011 Published by Elsevier Inc.

* Address reprint requests to Raymond Y. Kwong, MD, MPH, Director of Cardiac Magnetic Resonance Imaging, Assistant Professor of Medicine Brigham and Women's Hospital, Harvard Medical School, Boston, MA 02215. rykwong@ partners.org (R.Y. Kwong).

Statement of Conflict of Interest

All authors declare that there are no conflicts of interest. 
Noninvasive options available to assess patients suspected to have ischemia include exercise treadmill testing, nuclear myocardial perfusion imaging (MPI), positron emission tomography (PET), and stress echocardiography. Each has their own respective merits and technical limitations. The accuracy of nuclear scintigraphy may be limited by low spatial resolution and soft tissue attenuation artifacts. ${ }^{7,8}$ Stress echocardiography is less accurate in those with baseline wall motion abnormalities and left ventricular dysfunction. ${ }^{9,10}$ In addition, specific patient groups may pose additional challenges, such as persons with diabetes who have a propensity for diffuse coronary disease and women who require higher spatial resolution because of smaller heart size. ${ }^{11,12}$ The technical capabilities of cardiovascular magnetic resonance (CMR) can overcome some of these limitations. CMR is the current reference standard technique in assessing cardiac structure and function. In recent years, tissue characterization by CMR has shown strong association with patient prognosis. Likewise, ischemia assessment by stress CMR has undergone considerable technical advancement and now represents a safe, available, and potentially cost-effective clinical tool with both high diagnostic and prognostic accuracy. ${ }^{13}$

This review will discuss the utility of stress CMR for the assessment of myocardial ischemia and contrast and compare the relative attributes and limitations of CMR in comparison with other available modalities.

\section{Acute coronary syndromes}

Approximately 6 million patients in the United States present annually with chest pain to emergency departments (EDs) ${ }^{14}$ Although only a small fraction of these patients have a cardiac etiology for their pain, more than $50 \%$ of these patients will be admitted to a hospital, leading to $\$ 10$ to 12 billion in wasted expenditure per year. ${ }^{15} \mathrm{~A}$ significant source of this inefficiency stems from the difficulty physicians experience in diagnosing ACS in the ED, with $2 \%$ to $4 \%$ of patients with an ACS being inappropriately discharged from the ED. ${ }^{16}$ These factors underscore the need for novel diagnostic tests, such as CMR, that may be used to further investigate patients with suspicion of an ACS and who do not have electrocardiographic (ECG) findings diagnostic of ischemia or elevation of cardiac biomarkers.

Several clinical studies have investigated the diagnostic utility of CMR in urgent assessment of patients presenting with acute chest pain to the ED. In a single imaging session, CMR can assess for a range of abnormalities caused by an ACS, including wall motion abnormalities by cine imaging, abnormal coronary flow by resting first-pass perfusion deficits, acute myocardial edema by $\mathrm{T} 2$-weighted technique, or infarction by late gadolinium enhancement (LGE). The combined assessment of myocardial physiology and anatomical data, consisting of identification of the ischemic coronary territory and determination of the transmurality of infarction, by these CMR techniques forms a powerful tool in evaluating the spectrum of ACS for any given patient from threatened coronary occlusion without myocardial necrosis to frank infarction. ${ }^{17}$ In 1 prospective clinical study of 161 patients presenting to an ED with acute chest pain, Kwong et al ${ }^{18}$ reported a sensitivity of $84 \%$ and specificity of $85 \%$ for the detection of ACS based only on resting function and infarct detection using LGE-CMR. The diagnostic utilities of resting function and LGE were found to be complementary in this study, underscoring the importance of CMR capturing evidence of myocardial stunning and injury in coregistered scan planes within a single imaging session. Another study by Cury et $\mathrm{al}^{19}$ not only followed a similar imaging protocol but also included $\mathrm{T} 2$-weighted imaging. The results of this study provided strong evidence in support of the additional role of T2based edema imaging in differentiating acute from chronic infarction and thus enhancing the diagnostic specificity in detecting $\mathrm{ACS}^{20}$ as well as identifying the total area of myocardium at risk. ${ }^{19-21}$ 
The diagnostic performance of stress CMR was examined by Plein et $\mathrm{al}^{22}$ in 68 patients presenting to the ED with suspected ACS. For each patient, a comprehensive CMR assessment was performed assessing left ventricular function, rest and stress perfusion, LGE, and coronary magnetic resonance angiography. The comprehensive CMR analysis demonstrated a sensitivity of $96 \%$, with a specificity of $83 \%$ using quantitative coronary angiography (QCA) as the reference standard. The comprehensive analysis was more accurate than analysis of any individual CMR method..$^{22}$ Ingkanisorn et $\mathrm{al}^{23}$ evaluated the prognostic value of stress perfusion CMR in 135 patients presenting acutely to the ED with chest pain but no elevation of troponin I. Stress perfusion CMR had a sensitivity of $100 \%$ and specificity of $93 \%$ for detecting adverse cardiac outcomes at 1-year follow-up and was the most accurate predictor of adverse outcome in receiver operator characteristic (ROC) analysis. ${ }^{23}$ For those patients with infarction, resting perfusion CMR may reveal areas of microvascular obstruction within the infarct that have been shown to independently portend a worsened prognosis. ${ }^{24}$ Finally, CMR may be particularly helpful in the ED setting by excluding other etiologies of cardiac chest pain, such as myocarditis, pericardial disease, or stress-induced apical cardiomyopathy. ${ }^{25,26}$

With accumulating data regarding the high diagnostic accuracy of CMR for the assessment of acute chest pain, the cost-effectiveness of this approach was recently evaluated in a prospective, randomized, controlled trial comparing stress perfusion CMR to standard inpatient evaluation. The study population included 110 patients presenting to the ED with chest pain at intermediate or high probability for ACS, but without ECG or biomarker evidence of an MI. The patients assessed with CMR had significantly lower median hospitalization costs, and 79\% were managed without hospital admission. There were no adverse clinical outcomes at 30 days in either randomized groups, suggesting that CMR assessment in this setting was cost-effective. ${ }^{27}$

\section{Dobutamine stress CMR imaging}

Although exercise stress CMR may be performed with nonferromagnetic treadmill or supine bicycle testing, this approach is seldom used because of technical difficulties related to achieving target heart rate at the time of imaging. More commonly, pharmacologic stress CMR may be performed with dobutamine, an inotropic agent, or a vasodilator. The various vasodilators used include adenosine (adenoscan, generic), dipyridamole (persantine, generic), and regadenoson (lexiscan; Astellas Deerfield, Illinois) (Table 1). Recently, most high-volume institutions use regadenoson owing to its ease of administration as a single, small-volume intravenous bolus as opposed to an infusion.

Stress CMR has been shown to be extremely safe, ${ }^{28,29}$ with a comparable safety profile to nuclear $\mathrm{MPI}^{30}$ and dobutamine stress echo (DSE) ${ }^{26}$ Despite the inability to monitor STsegment changes at the time of stress because of the magnetohydrodynamic effect on ECG surface voltages, the development or worsening of regional wall motion abnormalities, which precede ECG changes, may be immediately detected. Furthermore, all patients undergo monitoring of heart rate and blood pressure. A physician skilled in the administration of cardiopulmonary resuscitation must always be present at all times during a stress CMR scan.

Stress CMR may be performed with 1.5 or $3.0 \mathrm{~T}$ scanning systems. Cine imaging is typically performed with steady-state free precession (SSFP) with acquisition accelerated by parallel imaging. Retrospective ECG gating is performed with multichannel ECG (vector ECG). Cine sequences typically have 20 to 30 phases per cardiac cycle with breath holds of 4 to 6 seconds for heart rates up to 200 beats per minute. In-plane spatial resolution is typically $1.5 \times 1.5 \mathrm{~mm}$ with a slice thickness of $8 \mathrm{~mm}$. The feasibility of stress CMR with 
real-time imaging, without the requirement for breath holding or a regular cardiac rhythm, has been demonstrated in a small study of 22 patients. ${ }^{17}$ Although a similar diagnostic accuracy was found to standard imaging, this method has both lower spatial and temporal resolution.

Dobutamine stress CMR (Ds-CMR) is performed with a similar protocol to DSE. Myocardial thickening and contraction of the 17-segment left ventricular model are evaluated at rest and while achieving $85 \%$ of age-predicted maximal heart rate (220-age) or higher to evaluate changes in contractility and wall thickening. ${ }^{31} \mathrm{~A}$ standard dosing regimen of $10,20,30$, and $40 \mu \mathrm{g} / \mathrm{kg}$ per minute of dobutamine is used, with increases at 3-minute intervals to attain target heart rate. Adjunctive atropine is administered for those patients who do not achieve target heart rate with dobutamine. Imaging of the left ventricle is performed in short-axis and radial long-axis views.

It has been reported that the sensitivity of Ds-CMR may be improved with the addition of myocardial tagging. Myocardial tagging permits the creation of either horizontal or vertical dark taglines or a grid over the myocardium by spatially selective saturation of the magnetization at the beginning of the cardiac cycle. The lines of saturated magnetizationappearing as dark lines-persist throughout the cardiac cycle as long as the heart interval duration is not much longer than the $\mathrm{T} 1$ relaxation time. The distortion of the tagging grid, for example, at end-systole, can arguably help to visually detect subtle wall motion abnormalities not readily apparent by visual estimation of wall thickening alone. Kuijpers et $\mathrm{al}^{31}$ demonstrated that the use of myocardial tagging improved the diagnostic accuracy of Ds-CMR as compared with standard SSFP imaging. Despite these promising results, the relatively small incremental value of tagging over visual interpretation, the additional efforts related to postprocessing tagged CMR cine studies, and the T1-dependent fading of myocardial tags over the duration of the cardiac cycle have curtailed more widespread uptake of this technique. More recently, however, tagging was used to evaluate ischemia by quantification of myocardial strain during intermediate stress. This approach demonstrated similar diagnostic accuracy to standard Ds-CMR and may potentially lead to even shorter and safer imaging protocols in the future ${ }^{33}$. The use of tagging may benefit from 3.0 T CMR where myocardial tagging can persist throughout the entire cardiac cycle as well as further automation of myocardial strain analysis with rapid display of strain maps.

Multiple studies have reported excellent diagnostic performance of Ds-CMR using QCA as the reference standard ${ }^{34}$ (Table 2).

Early studies demonstrated the feasibility of Ds-CMR and comparable diagnostic accuracy to nuclear perfusion imaging. ${ }^{35-37}$ However, these studies were limited based on their relatively small patient numbers and single-center experience. Two landmark studies in the 1990s helped to establish the clinical utility of Ds-CMR. The first by Nagel et al ${ }^{40}$ directly compared the diagnostic performance of Ds-CMR with DSE in 172 patients using QCA as the reference standard. They reported much higher diagnostic accuracy for Ds-CMR than DSE, which they largely attributed to superior image quality at peak stress with Ds-CMR. Almost $70 \%$ of Ds-CMR cases were graded as "good" image quality compared with less than $20 \%$ of DSE cases. Furthermore, less than 2\% of Ds-CMR were found to be "nondiagnostic," whereas almost 10\% of DSE cases could not be evaluated. The second study by Hundley et al ${ }^{41}$ evaluated the diagnostic accuracy of Ds-CMR in 153 patients with a nondiagnostic DSE and reported a sensitivity and specificity of $83 \%$. A recent metaanalysis pooling data from 735 patients reported a sensitivity of $83 \%$ and specificity of $86 \%$ for the detection of obstructive coronary stenosis greater than 50\% using QCA as the reference standard. ${ }^{46}$ Dobutamine stress CMR has also shown high reproducibility and low interobserver variability in a study of 150 cases read independently by 4 blinded readers. ${ }^{47}$ 
The diagnostic utility of combined wall motion and myocardial perfusion during peak dobutamine stress was evaluated in a study of 455 patients by Gebker et al. ${ }^{45}$ They demonstrated improved sensitivity ( $91 \%$ vs $85 \%, P=.001)$, but no difference in specificity for the detection of $70 \%$ or more obstructive coronary disease by QCA. Further work by this group demonstrated that the addition of first-pass perfusion imaging improved the diagnostic performance of Ds-CMR in patients with concentric left ventricular remodeling. ${ }^{48}$ Because of the presence of left ventricular hypertrophy, subtle regional wall motion abnormalities may be more difficult to interpret visually. The addition of first-pass perfusion imaging for these studies may be performed without significantly augmenting total examination duration.

Hundley et $\mathrm{al}^{49}$ evaluated the prognostic significance of Ds-CMR in a population of 279 patients with poor echocardiographic windows and found a significant, independent association with major adverse cardiovascular outcomes (MACE) and ischemia for patients with a left ventricular ejection fraction (LVEF) greater than $40 \%$. Single-center studies have demonstrated that an abnormal Ds-CMR portends a higher risk of cardiac death and MI independent of traditional risk factors. ${ }^{50,51}$ Conversely, patients with a normal Ds-CMR and LVEF of $60 \%$ or greater have shown an excellent prognosis with no events over a 2-year follow-up. More recently, larger studies, including 1 study with more than 1490 patients, reported an independent association with adverse cardiac outcomes in multivariate analysis for patients with an abnormal Ds-CMR ${ }^{52}$ and more than $99 \%$ event-free survival in patients with no evidence of ischemia over a 36 -month follow-up. ${ }^{53,54}$

Dobutamine stress CMR has also shown clinical utility for preoperative cardiac risk assessment before noncardiac surgery. In the subgroup of patients with intermediate clinical predictors of future cardiac events, a positive Ds-CMR proved to be an independent factor for predicting perioperative MI, cardiac death, or congestive heart failure ${ }^{55}$ Conversely, those patients with a normal Ds-CMR preoperatively had extremely low event rates. Dobutamine stress CMR has also demonstrated an independent association with adverse cardiac outcomes in 200 patients with baseline left ventricular dysfunction (LVEF $\mathbf{5 5 \%}$ ) followed up for a mean of 5 years. ${ }^{56}$

\section{Vasodilator stress CMR}

The initial vasodilators used for pharmacologic stress CMR were adenosine and dipyridamole (Table 1). Adenosine is an endogenous vasodilator that works via the adenosine 2 a receptor. Adenosine may induce wall motion abnormalities, which is specific but relatively insensitive for ischemia. Therefore, vasodilator stress CMR is used with perfusion imaging to denote relative perfusion defects on first-pass perfusion of the myocardium. ${ }^{42,57}$ Although most patients achieve an adequate hemodynamic response to standard adenosine dosing, high-dose adenosine infusions, to a maximum of $210 \mu \mathrm{g} / \mathrm{kg}$ per minute, has been shown to be both well tolerated and effective in those patients who do not achieve an adequate response with standard dosing. ${ }^{58}$ The specificity of perfusion abnormalities may be enhanced by adding LGE imaging to denote areas of prior infarction and rest perfusion to reduce artifacts. ${ }^{59}$

Cardiovascular magnetic resonance perfusion imaging is accomplished with the use of a contrast agent that permits visualization of the relative level and extent of perfusion between adjacent myocardial segments as well as a determination of the absolute perfusion of a particular segment. Gadolinium-based contrast agents (GBCAs) are highly attractive exogenous contrast agents owing to their relatively high T1 relaxivity due to 7 unpaired electrons and their safety profile in patients with normal or mildly impaired renal function. An intravenous GBCA bolus takes approximately 15 to 20 seconds for its first-pass 
perfusion through the cardiac chambers, and the flow-sensitive phase of myocardial contrast enhancement ("wash-in") occurs approximately within this time frame. Areas of perfused myocardium appear bright owing to GBCAs T1-shortening effects, whereas areas that are hypoperfused have lower signal intensity and appear dark (er). The relative intensity enhancement (eg, the rate of enhancement and maximal enhancement) may be contrasted to adjacent myocardial segments or by comparison of the contrast enhancement during vasodilation against its level at baseline (ie, during resting perfusion). This allows both estimated relative and absolute perfusion and also perfusion reserve, respectively (Fig 1).

Perfusion may be assessed qualitatively, semiquantitatively, or by fully quantitative methods. Signal-intensity changes measured during the first pass of contrast bolus through the myocardium may be quantitated by various methods, including the rate of contrast enhancement ("signal upslope"), the "upslope integral," calculated from the area under the upslope up to time point where contrast enhancement peaks in the blood pool, and the ratio of peak signal intensity normalized by baseline (ie, precontrast) signal intensity. ${ }^{60,61}$ Quantitative perfusion methods have been shown to vary inversely with the degree of coronary stenosis by QCA. ${ }^{62}$ For assessment of the perfusion reserve, a ratio of the myocardial upslope parameter values for rest and stress, normalized by the respective upslope of the signal intensity in the blood pool, is calculated to adjust for hemodynamic changes between rest and stress. More recently, anatomical assessment of perfusion as a criterion standard has come under criticism, with more physiologic invasive methods, such as fractional flow reserve (FFR), demonstrating not only more accurate flow assessment, but also improved clinical outcomes. ${ }^{63}$ Both quantitative and qualitative perfusions as assessed by CMR have shown high correlation with FFR using a cutoff value of less than $0.75 .{ }^{57,64}$ Studies have found that qualitative assessment of perfusion may be performed with a 0.1 $\mathrm{mmol} / \mathrm{kg}$ GBCA bolus to optimize visualization, ${ }^{65}$ whereas quantitative analysis was evaluated in a prospective, single-center trial, which found that $0.05 \mathrm{mmol} / \mathrm{kg}$ bolus maintained a linear relationship between signal from the blood pool and concentration of gadolinium. 66

Coronary flow reserve (CFR) may be estimated by CMR by calculating the difference in perfusion measured during stress and rest. ${ }^{67}$ Coronary flow reserve assessment was compared in 48 patients with coronary artery disease (CAD) and 18 healthy volunteers by dypridamole stress CMR. Using PET as the reference standard, CMR had a sensitivity and specificity of $91 \%$ and $94 \%$, respectively, for CAD detection in this study. ${ }^{34}$ Dypiridamole CMR has similar diagnostic performance to adenosine CMR and allows estimated CFR with comparable diagnostic accuracy to PET. ${ }^{34}$

Similar to Ds-CMR, stress perfusion CMR has also demonstrated excellent diagnostic performance for the detection of obstructive CAD (Table 3). Initial single-center studies reported both the feasibility of the technique as well as high diagnostic accuracy with QCA as the reference standard. Ishida et $\mathrm{al}^{68}$ examined 104 consecutive patients and found a sensitivity of $90 \%$ and specificity of $85 \%$ for coronary stenosis $50 \%$ or more. Nagel et al ${ }^{67}$ reported similar diagnostic performance in a study of 84 consecutive patients. After several single-center studies with similar findings, the utility of stress perfusion CMR was evaluated in several multicenter studies. Wolff et al ${ }^{66}$ performed a dose-ranging prospective multicenter study of gadolinium and reported an overall sensitivity of $93 \%$ and specificity of $75 \%$. In the same year, Giang et al ${ }^{65}$ reported similar diagnostic accuracy for a multicenter study of 94 patients evaluating a semiquantitative method of interpretation. These studies were followed by the much larger Comparison of Perfusion-cardiac Magnetic Resonance with Single-photon Emission Computed Tomography for the Detection of Coronary Artery Disease (MR-IMPACT) trial, ${ }^{74}$ a prospective, multicenter diagnostic performance study evaluating 234 patients in 18 centers with a standard CMR perfusion protocol using a 0.1 
$\mathrm{mmol} / \mathrm{kg}$ bolus dose of gadolinium designed to assess the real-world diagnostic performance of CMR perfusion compared with single-photon emission computed tomography (SPECT). The MR-IMPACT reported a superior diagnostic performance for stress CMR perfusion as compared with SPECT with QCA as the reference standard (ROC $86 \%$ vs $67 \%$ for nuclear MPI, $P=.01$ ). Subgroup analysis of patients with multivessel disease demonstrated even higher diagnostic performance for stress perfusion CMR as compared with nuclear MPI (ROC $89 \%$ vs $70 \%, P=.006$ ). In 2007, a meta-analysis pooled data from 24 studies with more than 1500 patients. They reported a sensitivity of $89 \%$, with a specificity of $80 \%$ for the detection of obstructive coronary stenosis greater than $50 \%$ in patients with high prevalence of CAD (57\%). ${ }^{46}$ More recently, a meta-analysis of more than 2100 patients from 26 studies demonstrated similar findings with a sensitivity of $89 \%$ and specificity of $80 \%$ for CAD prevalence of $57 \% .^{78}$

Myocardial perfusion imaging at 3.0 T may be advantageous for diagnostic assessment of ischemia because of superior signal to noise and contrast to noise ratios in addition to higher peak enhancement, which may allow greater differentiation between perfused and nonperfused myocardium as compared with $1.5 \mathrm{~T} .{ }^{79}$ Cheng et al ${ }^{80}$ compared the diagnostic performance of 3.0 vs $1.5 \mathrm{~T}$ scans in 61 patients with exertional chest pain using QCA as the diagnostic standard. They found the ROC analysis for $3.0 \mathrm{~T}$ demonstrated significantly higher area under the curve (AUC; 0.89 vs $0.70 ; P<.05$ ) for detecting coronary stenosis $50 \%$ or more. The sensitivity of $3.0 \mathrm{~T}$ was $98 \%$ vs $90 \%$ for $1.5 \mathrm{~T}$, with specificity of $76 \%$ compared with $67 \%$. CMR assessment by $3.0 \mathrm{~T}$ imaging does have limitations, including greater B1 inhomogeneity, which may compromise suppression of background tissues, higher $\mathrm{T} 2 *$ effects, which may require a reduction of gadolinium dose, and greater susceptibility to off- resonance artifacts, which may necessitate steps in adjusting imaging parameters and prolong scan times. Many of these issues can be resolved by performing shimming in improving the homogeneity of the magnetic field as well as modifications of pulse sequences and protocols. These factors, however, currently limit the use of 3.0 T CMR to more experienced centers.

Newer techniques focusing on enhanced spatial resolution with 3.0 T have also demonstrated clinical feasibility with stress perfusion imaging. ${ }^{81}$ Using acceleration techniques based on k-space and time sensitivity- encoded CMR, high spatial resolution scans may be performed for stress perfusion imaging. However, concerns regarding temporal blurring effects associated with higher acceleration factors have limited more widespread adoption of this technique. Manka et $\mathrm{al}^{81}$ evaluated a time sensitivity-encoded CMR method using parallel imaging resulting in 8-fold scan acceleration with in-plane spatial resolution during stress perfusion imaging of 1.1 by $1.1 \mathrm{~mm}^{2}$ in 20 patients referred for diagnostic angiography. Compared with QCA as the criterion standard, this method achieved a ROC curve with an AUC of 0.94 for detecting coronary stenosis of $50 \%$ or more. ${ }^{81}$ More recently, high spatial resolution stress perfusion imaging with $3.0 \mathrm{~T}$ was evaluated by comparison with FFR in 127 patients with known or suspected CAD. For a threshold FFR value less than 0.75, the ROC curve for high spatial resolution CMR had an AUC of $0.92(P<.0001)$ with a sensitivity of 0.82 and specificity of $0.94 .{ }^{64}$ Larger diagnostic performance studies may further improve the generalizability of high spatial resolution stress perfusion studies.

Stress perfusion CMR has shown strong prognostic association with adverse cardiac outcomes. From an acute perspective, the study already presented above in 135 patients presenting to the ED for evaluation of acute chest pain revealed no events in patients with normal stress perfusion CMR after 1 year of follow-up. ${ }^{23}$ Other studies found that stress perfusion CMR contributed incremental value over traditional risk factors in patients with stable chest pain. Pilz et al ${ }^{82}$ found that patients with a normal stress perfusion imaging had 
more than $99 \%$ freedom from MACE in 218 patients followed for 1 year. Steel et al ${ }^{83}$ evaluated the prognostic value of stress perfusion CMR and LGE in 254 patients with stable chest pain symptoms. They found a 3 -fold increased risk of cardiac death and MI for patients with ischemic deficits on stress perfusion CMR, which was the strongest variable in multivariate analysis of adverse outcome. Conversely, patients with no evidence of ischemia or LGE had more than $98 \%$ annual freedom from death or MI. ${ }^{83}$ Stress CMR has also demonstrated the ability to further prognosticate risk of future cardiac events in higher risk populations with established CAD. In a study of 513 patients who underwent both Ds-CMR and stress perfusion CMR with adenosine, the presence of ischemia by stress perfusion CMR was associated with a 12-fold increased risk of MACE after 2.3 mean years of followup. This was the only independent association in a multivariable analysis accounting for conventional coronary risk factors and resting left ventricular function. Dobutamine stress CMR demonstrated the same association with a 5-fold increased risk of MACE. ${ }^{44,54}$

Beyond ischemic assessment, CMR is capable of providing a quantitative, multicomponent examination that may be repeated serially to assess disease progression or response to therapy. The ability to characterize whether ischemic tissue is viable is an important component of the decision to subject patients to the risks of revascularization by percutaneous coronary intervention or coronary artery bypass grafting. The recently published Surgical Treatment for Ischemic Heart Failure trial ${ }^{84}$ underscored the difficulty in treating patients with an ischemic cardiomyopathy who are at much higher risk of perioperative mortality and surgical complications based on left ventricular dysfunction. ${ }^{85,86}$ However, within this higher risk cohort, revascularization of viable myocardium has been associated with functional recovery, ${ }^{87,88}$ prevention of adverse left ventricular remodeling, ${ }^{89}$ and reduction of MACE. ${ }^{84,90,91}$

\section{Cardiovascular magnetic resonance vs other modalities}

Stress CMR has several advantages over existing tests, including an excellent safety profile, high sensitivity, relative absence of artifacts induced by unfavorable body habitus, and superior spatial resolution. These technical factors permit ischemia assessment with high diagnostic accuracy in patients who may be challenging for other modalities. Standard techniques using SSFP imaging provides for a high signal-to-noise ratio with excellent contrast between myocardium and ventricular blood pool. In addition, the high spatial resolution of this technique provides outstanding endocardial border definition, without the need for any additional contrast agents, such as may be required with DSE. This provides ideal imaging conditions for assessment of inducible wall motion abnormalities that is prone to the visual limitations of DSE. Therefore, stress CMR may be used to assess patients with poor echocardiographic windows, such as obese patients, those with thoracic chest wall deformities, or low body mass index. ${ }^{40,41}$ Single-center studies have revealed superior diagnostic performance of stress CMR to DSE, ${ }^{40}$ particularly in those with resting wall motion abnormalities. ${ }^{43}$

Stress CMR has several advantages in comparison with nuclear MPI as well. Stress CMR is not limited by attenuation artifacts, does not use potentially harmful ionizing radiation, and has 3- to 4-fold higher spatial resolution than nuclear scintigraphy. A comprehensive CMR assessment is more time efficient than nuclear MPI, with the capability to perform stress and rest perfusion, cardiac function, and myocardial viability in 30 to 50 minutes, which compares favorably with the duration of a nuclear scintigraphy examination. Furthermore, stress CMR perfusion does not suffer from the plateau effects in first-pass extraction seen with commonly used radioactive nuclear tracers and, therefore, has been shown to characterize the magnitude of myocardial perfusion more completely than SPECT during maximal vasodilatation. ${ }^{61}$ The absence of harmful ionizing radiation allows serial stress 
CMR examinations that may be clinically required to reassess disease progression or to evaluate response to therapy. The high spatial resolution and endocardial border definition of stress CMR perfusion may allow appreciation of the extent and coronary distribution of the myocardial segments involved, thus providing evidence that allows differentiation of small vessel from epicardial coronary disease in patients presenting with chest pain. ${ }^{92,93}$ Patients with diffuse small vessel disease often have a concentric and circumferential distribution of perfusion defects during vasodilatation.

Stress CMR has demonstrated superior diagnostic performance to SPECT imaging and high correlation with PET perfusion studies. ${ }^{34}$ The MR-IMPACT trial described above found a significantly higher diagnostic performance for stress CMR as compared with SPECT imaging, particularly for patients with multivessel disease. ${ }^{74}$ Assessment of patients with multivessel disease is a known limitation to SPECT imaging because of the phenomenon of balanced ischemia. Larger studies of stress perfusion CMR have shown similar diagnostic performance to PET perfusion, with a sensitivity of $87 \%$ and specificity of $85 \% .{ }^{34}$ To address the diagnostic accuracy of stress CMR in comparison with SPECT imaging, the Clinical Evaluation of Magnetic Resonance Imaging in Coronary Heart Disease prospective clinical trial will evaluate 750 patients and monitor cardiac outcomes for 3 years. It will include prognostic and economic cost-effective analysis of the 2 modalities. ${ }^{94}$ The diagnostic performance results of this study were recently presented and reported an overall sensitivity and specificity of $86 \%$ and $83 \%$, respectively, for CMR compared with $67 \%$ and $83 \%$, respectively, for SPECT. The difference in sensitivity reached statistical significance. ${ }^{95}$ The forthcoming economic and prognostic data will provide important cost effectiveness data that will likely be integral to use of noninvasive diagnostic modalities in the future.

Stress CMR has demonstrated excellent diagnostic performance in patient subgroups that have shown limited diagnostic accuracy with alternative imaging modalities, including women. ${ }^{11,12}$ Women, on average, tend to have less typical symptoms of angina than men and are less amenable to diagnosis by exercise treadmill testing thus making non-imagingbased methods for assessing ischemia more challenging. SPECT imaging may be limited by breast attenuation artifact that can lead to false-positive tests and further unnecessary invasive imaging. Women also are exposed to substantially higher radiation than men when assessed by any cardiac imaging modality that involves the use of ionizing radiation. Likewise, DSE may also be limited because of poor acoustic windows in women with larger breasts that can obscure the apical views. Stress CMR, on the other hand, does not suffer from these limitations and may image the heart in any 2- or 3-dimensional plane.

Dobutamine stress CMR has shown similar diagnostic performance in women and men. Gebker et al ${ }^{48}$ recently found similar diagnostic performance for Ds-CMR in 745 consecutive patients (204 women and $541 \mathrm{men}$ ) and furthermore reported no difference in regional accuracy for all 3 coronary vascular territories in patients with single-vessel disease. Dobutamine stress CMR has also shown significant prognostic value in women with a 4-fold increased risk of cardiac death and MI in women with an inducible wall motion abnormality vs those with no evidence of ischemia. ${ }^{96}$ In a recent study, our group found that CMR stress perfusion could be an effective method in assessing and prognosticating women suspected to have ischemia without the use of any ionizing radiation. (Filho et al, in press, 2011). Filho et al assessed 424 patients with intermediate risk of CAD and found that the annual combined event rate of cardiac death or acute MI was low at $0.3 \%$ in women without evidence of ischemia, which compared with a high annual event rate of $15 \%$ in those with evidence of ischemia.

Another significant advantage of stress CMR is the capability for highly accurate adjunctive myocardial viability assessment, particularly useful in patients being evaluated for an 
ischemic cardiomyopathy. The coupling of physiologic myocardial perfusion with anatomical information regarding transmural extent of myocardial scar by LGE can aid in establishing not only the extent of ischemia, but also quantitating the relative proportion of hibernating myocardium that may potentially benefit from revascularization. In addition, Ds-CMR has shown excellent diagnostic performance for prediction of functional recovery after coronary revascularization. ${ }^{50,97}$ This method can complement standard viability assessment by LGE to evaluate the extent of myocardial scar.

The presence of LGE as detected by CMR provides evidence of prior infarction and powerful prognostic data. Late gadolinium enhancement has been shown to be the strongest predictor of subsequent cardiac events in 2 studies with collectively more than 1000 patients with suspected CAD, but no known ischemic heart disease. ${ }^{98,99}$ Late gadolinium enhancement was the strongest multivariable predictor while taking into account conventional clinical, angiographic, and echocardiographic risk factors. In addition to regional assessment of fibrosis, novel T1 mapping techniques with CMR allow quantitative assessment of diffuse myocardial fibrosis from accumulation of collagen and extracellular material. ${ }^{100}$ Diffuse fibrosis may result from ischemic or hypertensive heart disease, among other cardiac conditions, and increase propensity for arrhythmias and both diastolic and systolic dysfunction. ${ }^{101,102}$ Our group has recently demonstrated a method for assessing the extent of extracellular matrix remodeling through quantification of the extracellular volume fraction, termed the fibrosis index. ${ }^{103}$ These promising techniques may elucidate powerful diagnostic and prognostic markers in patients with CAD. Standard CMR assessment also provides for highly accurate structural and functional assessment now considered the criterion standard. This additional data may be quite helpful and limit further diagnostic testing in patients undergoing ischemic assessment, which may be required with other modalities that are limited to ischemic assessment alone.

\section{Limitations of CMR}

Cardiovascular magnetic resonance has several limitations that currently restrict its utility for assessing patients with possible ischemia. The usual list of biomedical metallic devices that represent contraindications to magnetic resonance imaging still applies, although a pacemaker model, compatible with magnetic resonance imaging at $1.5 \mathrm{~T}$, is now Food and Drug Administration approved and commercially available. ${ }^{104}$ Stress CMR perfusion imaging for clinical assessment currently involves the use of GBCAs that have been associated with a serious complication known as nephrogenic systemic fibrosis (NSF). ${ }^{105}$ Since the original description of this association in 2006, it is now known that NSF is a rare complication that occurs primarily in patients with end-stage renal disease or rapidly deteriorating renal function and those with concurrent acute illnesses. ${ }^{106,107}$ The risk of NSF appears to be substantially lower with newer cyclic-structured gadolinium compounds. Most institutions now use serum estimated glomerular filtration rate in at-risk patients and either perform imaging studies with reduced contrast dose or avoid the use of gadolinium altogether. As a result, new cases of NSF from gadolinium exposure have been extremely rare in recent years.

\section{Conclusion}

Cardiovascular magnetic resonance can provide comprehensive diagnostic and prognostic assessment of myocardial ischemia for patients presenting with chest pain, stable angina, or for myocardial viability. Stress CMR is a highly accurate and safe diagnostic modality that has widespread patient applicability and has proven advantageous for certain patient subgroups, including those with poor echocardiographic windows, baseline left ventricular dysfunction, and resting wall motion abnormalities. The high temporal and spatial resolution 
of stress CMR permits not only accurate identification of CAD, but also the extent of myocardial ischemia and has shown to be a powerful prognostic marker of adverse cardiac events. Future cost-effective analysis may reveal more widespread indications to use stress CMR as a first-line diagnostic modality for myocardial ischemia assessment.

\section{Abbreviations and Acronyms}

ACS

AUC

CAD

CFR

CMR

Ds-CMR

DSE

ECG

ED

FFR

GBCA

LGE

LVEF

MACE

MI

MPI

MR-IMPACT

NSF

PET

QCA

ROC

SPECT

SSFP acute coronary syndrome

area under the curve

coronary artery disease

coronary flow reserve

cardiovascular magnetic resonance

dobutamine stress cardiovascular magnetic resonance

dobutamine stress echo

electrocardiographic

emergency department

fractional flow reserve

gadolinium-based contrast agent

late gadolinium enhancement

left ventricular ejection fraction

major adverse cardiovascular outcomes

myocardial infarction

myocardial perfusion imaging

Comparison of Perfusion-cardiac Magnetic Resonance with Singlephoton Emission Computed Tomography for the Detection of Coronary Artery Disease

nephrogenic systemic fibrosis

positron emission tomography

quantitative coronary angiography

receiver operator characteristic

single-photon emission computed tomography

steady-state free precession

\section{References}

1. Watkins S, Thiemann D, Coresh J, et al. Fourteen-year (1987 to 2000) trends in the attack rates of, therapy for, and mortality from non-ST-elevation acute coronary syndromes in four United States communities. Am J Cardiol. 2005; 96:1349-1355. [PubMed: 16275176]

2. Thom T, Haase N, Rosamond W, et al. Heart disease and stroke statistics-2006 update: a report from the American Heart Association Statistics Committee and Stroke Statistics Subcommittee. Circulation. 2006; 113:e85-e151. [PubMed: 16407573]

3. Yao SS, Rozanski A. Principal uses of myocardial perfusion scintigraphy in the management of patients with known or suspected coronary artery disease. Prog Cardiovasc Dis. 2001; 43:281-302. [PubMed: 11235845] 
4. Vanzetto G, Ormezzano O, Fagret D, et al. Long-term additive prognostic value of thallium-201 myocardial perfusion imaging over clinical and exercise stress test in low to intermediate risk patients : study in 1137 patients with 6-year follow-up. Circulation. 1999; 100:1521-1527. [PubMed: 10510055]

5. Hachamovitch R, Berman DS, Shaw LJ, et al. Incremental prognostic value of myocardial perfusion single photon emission computed tomography for the prediction of cardiac death: differential stratification for risk of cardiac death and myocardial infarction. Circulation. 1998; 97:535-543. [PubMed: 9494023]

6. Hachamovitch R, Berman DS, Kiat H, et al. Value of stress myocardial perfusion single photon emission computed tomography in patients with normal resting electrocardiograms: an evaluation of incremental prognostic value and cost-effectiveness. Circulation. 2002; 105:823-829. [PubMed: 11854122]

7. Krenning BJ, Geleijnse ML, Poldermans D, et al. Methodological analysis of diagnostic dobutamine stress echocardiography studies. Echocardiography. 2004; 21:725-736. [PubMed: 15546374]

8. Freedman N, Schechter D, Klein M, et al. SPECT attenuation artifacts in normal and overweight persons: insights from a retrospective comparison of $\mathrm{Rb}-82$ positron emission tomography and TI-201 SPECT myocardial perfusion imaging. Clin Nucl Med. 2000; 25:1019-1023. [PubMed: 11129138]

9. Bach DS, Muller DW, Gros BJ, et al. False positive dobutamine stress echocardiograms: characterization of clinical, echocardiographic and angiographic findings. J Am Coll Cardiol. 1994; 24:928-933. [PubMed: 7930226]

10. Ha JW, Juracan EM, Mahoney DW, et al. Hypertensive response to exercise: a potential cause for new wall motion abnormality in the absence of coronary artery disease. J Am Coll Cardiol. 2002; 39:323-327. [PubMed: 11788226]

11. Smart SC, Bhatia A, Hellman R, et al. Dobutamine-atropine stress echocardiography and dipyridamole sestamibi scintigraphy for the detection of coronary artery disease: limitations and concordance. J Am Coll Cardiol. 2000; 36:1265-1273. [PubMed: 11028482]

12. Hansen CL, Crabbe D, Rubin S. Lower diagnostic accuracy of thallium-201 SPECT myocardial perfusion imaging in women: an effect of smaller chamber size. J Am Coll Cardiol. 1996; 28:1214-1219. [PubMed: 8890818]

13. Karamitsos TD, Dall'armellina E, Choudhury RP, et al. Ischemic heart disease: comprehensive evaluation by cardiovascular magnetic resonance. Am Heart J. 2011; 162:16-30. [PubMed: 21742086]

14. McCaig LF, Nawar EW. National Hospital Ambulatory Medical Care Survey: 2004 emergency department summary. Adv Data. 2006:1-29.

15. Farkouh ME, Smars PA, Reeder GS, et al. A clinical trial of a chest-pain observation unit for patients with unstable angina. Chest Pain Evaluation in the Emergency Room (CHEER) Investigators. N Engl J Med. 1998; 339:1882-1888. [PubMed: 9862943]

16. Pope JH, Aufderheide TP, Ruthazer R, et al. Missed diagnoses of acute cardiac ischemia in the emergency department. N Engl J Med. 2000; 342:1163-1170. [PubMed: 10770981]

17. Schalla S, Klein C, Paetsch I, et al. Real-time MR image acquisition during high-dose dobutamine hydrochloride stress for detecting left ventricular wall-motion abnormalities in patients with coronary arterial disease. Radiology. 2002; 224:845-851. [PubMed: 12202724]

18. Kwong RY, Schussheim AE, Rekhraj S, et al. Detecting acute coronary syndrome in the emergency department with cardiac magnetic resonance imaging. Circulation. 2003; 107:531-537. [PubMed: 12566362]

19. Cury RC, Shash K, Nagurney JT, et al. Cardiac magnetic resonance with T2-weighted imaging improves detection of patients with acute coronary syndrome in the emergency department. Circulation. 2008; 118:837-844. [PubMed: 18678772]

20. Abdel-Aty H, Zagrosek A, Schulz-Menger J, et al. Delayed enhancement and T2-weighted cardiovascular magnetic resonance imaging differentiate acute from chronic myocardial infarction. Circulation. 2004; 109:2411-2416. [PubMed: 15123531]

21. Aletras AH, Tilak GS, Natanzon A, et al. Retrospective determination of the area at risk for reperfused acute myocardial infarction with T2-weighted cardiac magnetic resonance imaging:

Prog Cardiovasc Dis. Author manuscript; available in PMC 2014 March 14. 
histopathological and displacement encoding with stimulated echoes (DENSE) functional validations. Circulation. 2006; 113:1865-1870. [PubMed: 16606793]

22. Plein S, Greenwood JP, Ridgway JP, et al. Assessment of non-ST-segment elevation acute coronary syndromes with cardiac magnetic resonance imaging. J Am Coll Cardiol. 2004; 44:21732181. [PubMed: 15582315]

23. Ingkanisorn WP, Kwong RY, Bohme NS, et al. Prognosis of negative adenosine stress magnetic resonance in patients presenting to an emergency department with chest pain. J Am Coll Cardiol. 2006; 47:1427-1432. [PubMed: 16580532]

24. Wu KC, Zerhouni EA, Judd RM, et al. Prognostic significance of microvascular obstruction by magnetic resonance imaging in patients with acute myocardial infarction. Circulation. 1998; 97:765-772. [PubMed: 9498540]

25. Assomull RG, Lyne JC, Keenan N, et al. The role of cardiovascular magnetic resonance in patients presenting with chest pain, raised troponin, and unobstructed coronary arteries. Eur Heart J. 2007; 28:1242-1249. [PubMed: 17478458]

26. Codreanu A, Djaballah W, Angioi M, et al. Detection of myocarditis by contrast-enhanced MRI in patients presenting with acute coronary syndrome but no coronary stenosis. J Magn Reson Imaging. 2007; 25:957-964. [PubMed: 17457796]

27. Miller CD, Hwang W, Hoekstra JW, et al. Stress cardiac magnetic resonance imaging with observation unit care reduces cost for patients with emergent chest pain: a randomized trial. Ann Emerg Med. 2010; 56:209-219. e2. [PubMed: 20554078]

28. Wahl A, Paetsch I, Gollesch A, et al. Safety and feasibility of high-dose dobutamine-atropine stress cardiovascular magnetic resonance for diagnosis of myocardial ischaemia: experience in 1000 consecutive cases. Eur Heart J. 2004; 25:1230-1236. [PubMed: 15246641]

29. Kuijpers D, Janssen CH, van Dijkman PR, et al. Dobutamine stress MRI. Part I. Safety and feasibility of dobutamine cardiovascular magnetic resonance in patients suspected of myocardial ischemia. Eur Radiol. 2004; 14:1823-1828. [PubMed: 15278415]

30. Shah DJ, Kim HW, Kim RJ. Evaluation of ischemic heart disease. Heart Fail Clin. 2009; 5:315332. [PubMed: 19564011]

31. Kuijpers D, Ho KY, van Dijkman PR, et al. Dobutamine cardiovascular magnetic resonance for the detection of myocardial ischemia with the use of myocardial tagging. Circulation. 2003; 107:1592-1597. [PubMed: 12668491]

32. Zerhouni EA, Parish DM, Rogers WJ, et al. Human heart: tagging with MR imaging — a method for noninvasive assessment of myocardial motion. Radiology. 1988; 169:59-63. [PubMed: 3420283]

33. Korosoglou G, Lehrke S, Wochele A, et al. Strain-encoded CMR for the detection of inducible ischemia during intermediate stress. JACC Cardiovasc Imaging. 2010; 3:361-371. [PubMed: 20394897]

34. Schwitter J, Nanz D, Kneifel S, et al. Assessment of myocardial perfusion in coronary artery disease by magnetic resonance: a comparison with positron emission tomography and coronary angiography. Circulation. 2001; 103:2230-2235. [PubMed: 11342469]

35. Pennell DJ, Underwood SR, Ell PJ, et al. Dipyridamole magnetic resonance imaging: a comparison with thallium-201 emission tomography. Br Heart J. 1990; 64:362-369. [PubMed: 2271342]

36. Pennell DJ, Underwood SR, Manzara CC, et al. Magnetic resonance imaging during dobutamine stress in coronary artery disease. Am J Cardiol. 1992; 70:34-40. [PubMed: 1615867]

37. van Rugge FP, van der Wall EE, de Roos A, et al. Dobutamine stress magnetic resonance imaging for detection of coronary artery disease. J Am Coll Cardiol. 1993; 22:431-439. [PubMed: 8335812]

38. Baer FM, Voth E, Theissen P, et al. Coronary artery disease: findings with GRE MR imaging and Tc-99 m-methoxyisobutyl-isonitrile SPECT during simultaneous dobutamine stress. Radiology. 1994; 193:203-209. [PubMed: 8090892]

39. van Rugge FP, van der Wall EE, Spanjersberg SJ, et al. Magnetic resonance imaging during dobutamine stress for detection and localization of coronary artery disease. Quantitative wall motion analysis using a modification of the centerline method. Circulation. 1994; 90:127-138. [PubMed: 8025988] 
40. Nagel E, Lehmkuhl HB, Bocksch W, et al. Noninvasive diagnosis of ischemia-induced wall motion abnormalities with the use of high- dose dobutamine stress MRI: comparison with dobutamine stress echocardiography. Circulation. 1999; 99:763-770. [PubMed: 9989961]

41. Hundley WG, Hamilton CA, Thomas MS, et al. Utility of fast cine magnetic resonance imaging and display for the detection of myocardial ischemia in patients not well suited for second harmonic stress echocardiography. Circulation. 1999; 100:1697-1702. [PubMed: 10525488]

42. Paetsch I, Jahnke C, Wahl A, et al. Comparison of dobutamine stress magnetic resonance, adenosine stress magnetic resonance, and adenosine stress magnetic resonance perfusion. Circulation. 2004; 110:835-842. [PubMed: 15289384]

43. Wahl A, Paetsch I, Roethemeyer S, et al. High-dose dobutamine-atropine stress cardiovascular MR imaging after coronary revascularization in patients with wall motion abnormalities at rest. Radiology. 2004; 233:210-216. [PubMed: 15304662]

44. Jahnke C, Paetsch I, Gebker R, et al. Accelerated 4D dobutamine stress MR imaging with k-t BLAST: feasibility and diagnostic performance. Radiology. 2006; 241:718-728. [PubMed: 17065561]

45. Gebker R, Jahnke C, Manka R, et al. Additional value of myocardial perfusion imaging during dobutamine stress magnetic resonance for the assessment of coronary artery disease. Circ Cardiovasc Imaging. 2008; 1:122-130. [PubMed: 19808529]

46. Nandalur KR, Dwamena BA, Choudhri AF, et al. Diagnostic performance of stress cardiac magnetic resonance imaging in the detection of coronary artery disease: a meta-analysis. $\mathrm{J}$ Am Coll Cardiol. 2007; 50:1343-1353. [PubMed: 17903634]

47. Paetsch I, Jahnke C, Ferrari VA, et al. Determination of interobserver variability for identifying inducible left ventricular wall motion abnormalities during dobutamine stress magnetic resonance imaging. Eur Heart J. 2006; 27:1459-1464. [PubMed: 16613929]

48. Gebker R, Mirelis JG, Jahnke C, et al. Influence of left ventricular hypertrophy and geometry on diagnostic accuracy of wall motion and perfusion magnetic resonance during dobutamine stress. Circ Cardiovasc Imaging. 2010; 3:507-514. [PubMed: 20576810]

49. Hundley WG, Morgan TM, Neagle CM, et al. Magnetic resonance imaging determination of cardiac prognosis. Circulation. 2002; 106:2328-2333. [PubMed: 12403662]

50. Baer FM, Theissen P, Schneider CA, et al. Dobutamine magnetic resonance imaging predicts contractile recovery of chronically dysfunctional myocardium after successful revascularization. J Am Coll Cardiol. 1998; 31:1040-1048. [PubMed: 9562005]

51. Bodi V, Sanchis J, Lopez-Lereu MP, et al. Prognostic value of dipyridamole stress cardiovascular magnetic resonance imaging in patients with known or suspected coronary artery disease. $\mathrm{J}$ Am Coll Cardiol. 2007; 50:1174-1179. [PubMed: 17868810]

52. Korosoglou G, Elhmidi Y, Steen H, et al. Prognostic value of high-dose dobutamine stress magnetic resonance imaging in 1,493 consecutive patients: assessment of myocardial wall motion and perfusion. J Am Coll Cardiol. 2010; 56:1225-1234. [PubMed: 20883929]

53. Kuijpers D, van Dijkman PR, Janssen CH, et al. Dobutamine stress MRI. Part II. Risk stratification with dobutamine cardiovascular magnetic resonance in patients suspected of myocardial ischemia. Eur Radiol. 2004; 14:2046-2052. [PubMed: 15278416]

54. Jahnke C, Nagel E, Gebker R, et al. Prognostic value of cardiac magnetic resonance stress tests: adenosine stress perfusion and dobutamine stress wall motion imaging. Circulation. 2007; 115:1769-1776. [PubMed: 17353441]

55. Rerkpattanapipat $\mathrm{P}$, Morgan TM, Neagle CM, et al. Assessment of preoperative cardiac risk with magnetic resonance imaging. Am J Cardiol. 2002; 90:416-419. [PubMed: 12161234]

56. Dall'Armellina E, Morgan TM, Mandapaka S, et al. Prediction of cardiac events in patients with reduced left ventricular ejection fraction with dobutamine cardiovascular magnetic resonance assessment of wall motion score index. J Am Coll Cardiol. 2008; 52:279-286. [PubMed: 18634983]

57. Rieber J, Huber A, Erhard I, et al. Cardiac magnetic resonance perfusion imaging for the functional assessment of coronary artery disease: a comparison with coronary angiography and fractional flow reserve. Eur Heart J. 2006; 27:1465-1471. [PubMed: 16720685] 
58. Karamitsos TD, Ntusi NA, Francis JM, et al. Feasibility and safety of high-dose adenosine perfusion cardiovascular magnetic resonance. J Cardiovasc Magn Reson. 2010; 12:66. [PubMed: 21080924]

59. Klem I, Heitner JF, Shah DJ, et al. Improved detection of coronary artery disease by stress perfusion cardiovascular magnetic resonance with the use of delayed enhancement infarction imaging. J Am Coll Cardiol. 2006; 47:1630-1638. [PubMed: 16631001]

60. Klocke FJ, Simonetti OP, Judd RM, et al. Limits of detection of regional differences in vasodilated flow in viable myocardium by first-pass magnetic resonance perfusion imaging. Circulation. 2001; 104:2412-2416. [PubMed: 11705817]

61. Lee DC, Simonetti OP, Harris KR, et al. Magnetic resonance versus radionuclide pharmacological stress perfusion imaging for flow-limiting stenoses of varying severity. Circulation. 2004; 110:5865. [PubMed: 15210596]

62. Cullen JH, Horsfield MA, Reek CR, et al. A myocardial perfusion reserve index in humans using first-pass contrast-enhanced magnetic resonance imaging. J Am Coll Cardiol. 1999; 33:13861394. [PubMed: 10193743]

63. Tonino PA, De Bruyne B, Pijls NH, et al. Fractional flow reserve versus angiography for guiding percutaneous coronary intervention. N Engl J Med. 2009; 360:213-224. [PubMed: 19144937]

64. Lockie T, Ishida M, Perera D, et al. High-resolution magnetic resonance myocardial perfusion imaging at 3.0-Tesla to detect hemodynamically significant coronary stenoses as determined by fractional flow reserve. J Am Coll Cardiol. 2011; 57:70-75. [PubMed: 21185504]

65. Giang TH, Nanz D, Coulden R, et al. Detection of coronary artery disease by magnetic resonance myocardial perfusion imaging with various contrast medium doses: first European multi-centre experience. Eur Heart J. 2004; 25:1657-1665. [PubMed: 15351166]

66. Wolff SD, Schwitter J, Coulden R, et al. Myocardial first-pass perfusion magnetic resonance imaging: a multicenter dose-ranging study. Circulation. 2004; 110:732-737. [PubMed: 15289374]

67. Nagel E, Klein C, Paetsch I, et al. Magnetic resonance perfusion measurements for the noninvasive detection of coronary artery disease. Circulation. 2003; 108:432-437. [PubMed: 12860910]

68. Ishida N, Sakuma H, Motoyasu M, et al. Noninfarcted myocardium: correlation between dynamic first-pass contrast-enhanced myocardial MR imaging and quantitative coronary angiography. Radiology. 2003; 229:209-216. [PubMed: 12944596]

69. Doyle M, Fuisz A, Kortright E, et al. The impact of myocardial flow reserve on the detection of coronary artery disease by perfusion imaging methods: an NHLBI WISE study. J Cardiovasc Magn Reson. 2003; 5:475-485. [PubMed: 12882078]

70. Takase B, Nagata M, Kihara T, et al. Whole-heart dipyridamole stress first-pass myocardial perfusion MRI for the detection of coronary artery disease. Jpn Heart J. 2004; 45:475-486. [PubMed: 15240967]

71. Plein S, Radjenovic A, Ridgway JP, et al. Coronary artery disease: myocardial perfusion MR imaging with sensitivity encoding versus conventional angiography. Radiology. 2005; 235:423430. [PubMed: 15858084]

72. Pilz G, Bernhardt P, Klos M, et al. Clinical implication of adenosine-stress cardiac magnetic resonance imaging as potential gatekeeper prior to invasive examination in patients with AHA/ ACC class II indication for coronary angiography. Clin Res Cardiol. 2006; 95:531-538. [PubMed: 16897145]

73. Merkle N, Wohrle J, Grebe O, et al. Assessment of myocardial perfusion for detection of coronary artery stenoses by steady-state, free-precession magnetic resonance first-pass imaging. Heart. 2007; 93:1381-1385. [PubMed: 17488772]

74. Schwitter J, Wacker CM, van Rossum AC, et al. MR-IMPACT: comparison of perfusion-cardiac magnetic resonance with single-photon emission computed tomography for the detection of coronary artery disease in a multicentre, multivendor, randomized trial. Eur Heart J. 2008; 29:480489. [PubMed: 18208849]

75. Gebker R, Jahnke C, Paetsch I, et al. Diagnostic performance of myocardial perfusion MR at $3 \mathrm{~T}$ in patients with coronary artery disease. Radiology. 2008; 247:57-63. [PubMed: 18305188]

Prog Cardiovasc Dis. Author manuscript; available in PMC 2014 March 14. 
76. Klem I, Greulich S, Heitner JF, et al. Value of cardiovascular magnetic resonance stress perfusion testing for the detection of coronary artery disease in women. JACC Cardiovasc Imaging. 2008; 1:436-445. [PubMed: 19356464]

77. Thomas D, Strach K, Meyer C, et al. Combined myocardial stress perfusion imaging and myocardial stress tagging for detection of coronary artery disease at 3 Tesla. J Cardiovasc Magn Reson. 2008; 10:59. [PubMed: 19094196]

78. Hamon M, Fau G, Nee G, et al. Meta-analysis of the diagnostic performance of stress perfusion cardiovascular magnetic resonance for detection of coronary artery disease. J Cardiovasc Magn Reson. 2010; 12:29. [PubMed: 20482819]

79. Araoz PA, Glockner JF, McGee KP, et al. 3 Tesla MR imaging provides improved contrast in firstpass myocardial perfusion imaging over a range of gadolinium doses. J Cardiovasc Magn Reson. 2005; 7:559-564. [PubMed: 15959968]

80. Cheng AS, Pegg TJ, Karamitsos TD, et al. Cardiovascular magnetic resonance perfusion imaging at 3-tesla for the detection of coronary artery disease: a comparison with 1.5-tesla. J Am Coll Cardiol. 2007; 49:2440-2449. [PubMed: 17599608]

81. Manka R, Vitanis V, Boesiger P, et al. Clinical feasibility of accelerated, high spatial resolution myocardial perfusion imaging. JACC Cardiovasc Imaging. 2010; 3:710-717. [PubMed: 20633848]

82. Pilz G, Jeske A, Klos M, et al. Prognostic value of normal adenosine-stress cardiac magnetic resonance imaging. Am J Cardiol. 2008; 101:1408-1412. [PubMed: 18471450]

83. Steel K, Broderick R, Gandla V, et al. Complementary prognostic values of stress myocardial perfusion and late gadolinium enhancement imaging by cardiac magnetic resonance in patients with known or suspected coronary artery disease. Circulation. 2009; 120:1390-1400. [PubMed: 19770399]

84. Velazquez EJ, Lee KL, Deja MA, et al. Coronary-artery bypass surgery in patients with left ventricular dysfunction. N Engl J Med. 2011; 364:1607-1616. [PubMed: 21463150]

85. Christakis GT, Weisel RD, Fremes SE, et al. Coronary artery bypass grafting in patients with poor ventricular function. Cardiovascular Surgeons of the University of Toronto. J Thorac Cardiovasc Surg. 1992; 103:1083-1091. [discussion 91-2]. [PubMed: 1597972]

86. Hochberg MS, Parsonnet V, Gielchinsky I, et al. Coronary artery bypass grafting in patients with ejection fractions below forty percent. Early and late results in 466 patients. J Thorac Cardiovasc Surg. 1983; 86:519-527. [PubMed: 6604845]

87. van den Berg EK Jr, Popma JJ, Dehmer GJ, et al. Reversible segmental left ventricular dysfunction after coronary angioplasty. Circulation. 1990; 81:1210-1216. [PubMed: 2317903]

88. Ragosta M, Beller GA, Watson DD, et al. Quantitative planar rest-redistribution 201Tl imaging in detection of myocardial viability and prediction of improvement in left ventricular function after coronary bypass surgery in patients with severely depressed left ventricular function. Circulation. 1993; 87:1630-1641. [PubMed: 8491019]

89. Carluccio E, Biagioli P, Alunni G, et al. Patients with hibernating myocardium show altered left ventricular volumes and shape, which revert after revascularization: evidence that dyssynergy might directly induce cardiac remodeling. J Am Coll Cardiol. 2006; 47:969-977. [PubMed: 16516079]

90. Alderman EL, Fisher LD, Litwin P, et al. Results of coronary artery surgery in patients with poor left ventricular function (CASS). Circulation. 1983; 68:785-795. [PubMed: 6352078]

91. Allman KC, Shaw LJ, Hachamovitch R, et al. Myocardial viability testing and impact of revascularization on prognosis in patients with coronary artery disease and left ventricular dysfunction: a meta-analysis. J Am Coll Cardiol. 2002; 39:1151-1158. [PubMed: 11923039]

92. Panting JR, Gatehouse PD, Yang GZ, et al. Abnormal subendocardial perfusion in cardiac syndrome $\times$ detected by cardiovascular magnetic resonance imaging. N Engl J Med. 2002; 346:1948-1953. [PubMed: 12075055]

93. Keijer JT, van Rossum AC, Wilke N, et al. Magnetic resonance imaging of myocardial perfusion in single-vessel coronary artery disease: implications for transmural assessment of myocardial perfusion. J Cardiovasc Magn Reson. 2000; 2:189-200. [PubMed: 11545116] 
94. Greenwood JP, Maredia N, Radjenovic A, et al. Clinical evaluation of magnetic resonance imaging in coronary heart disease: the CE-MARC study. Trials. 2009; 10:62. [PubMed: 19640271]

95. Greenwood JP, Maredia N, Younger JF, et al. Abstract 21797: Clinical Evaluation of Magnetic Resonance Imaging in Coronary Heart Disease (The CE-MARC Study): a prospective evaluation of 750 patients. Circulation. 2010; 122:A21797.

96. Wallace EL, Morgan TM, Walsh TF, et al. Dobutamine cardiac magnetic resonance results predict cardiac prognosis in women with known or suspected ischemic heart disease. JACC Cardiovasc Imaging. 2009; 2:299-307. [PubMed: 19356575]

97. Wellnhofer E, Olariu A, Klein C, et al. Magnetic resonance low-dose dobutamine test is superior to SCAR quantification for the prediction of functional recovery. Circulation. 2004; 109:2172-2174. [PubMed: 15117834]

98. Cheong BY, Muthupillai R, Wilson JM, et al. Prognostic significance of delayed-enhancement magnetic resonance imaging: survival of 857 patients with and without left ventricular dysfunction. Circulation. 2009; 120:2069-2076. [PubMed: 19901193]

99. Kwong RY, Chan AK, Brown KA, et al. Impact of unrecognized myocardial scar detected by cardiac magnetic resonance imaging on event-free survival in patients presenting with signs or symptoms of coronary artery disease. Circulation. 2006; 113:2733-2743. [PubMed: 16754804]

100. Iles L, Pfluger H, Phrommintikul A, et al. Evaluation of diffuse myocardial fibrosis in heart failure with cardiac magnetic resonance contrast-enhanced T1 mapping. J Am Coll Cardiol. 2008; 52:1574-1580. [PubMed: 19007595]

101. Kawara T, Derksen R, de Groot JR, et al. Activation delay after premature stimulation in chronically diseased human myocardium relates to the architecture of interstitial fibrosis. Circulation. 2001; 104:3069-3075. [PubMed: 11748102]

102. Weber KT, Brilla CG. Pathological hypertrophy and cardiac interstitium. Fibrosis and reninangiotensin-aldosterone system. Circulation. 1991; 83:1849-1865. [PubMed: 1828192]

103. Kehr E, Sono M, Chugh SS, et al. Gadolinium-enhanced magnetic resonance imaging for detection and quantification of fibrosis in human myocardium in vitro. Int J Cardiovasc Imaging. 2008; 24:61-68. [PubMed: 17429755]

104. Wilkoff BL, Bello D, Taborsky M, et al. Magnetic resonance imaging in patients with a pacemaker system designed for the magnetic resonance environment. Heart Rhythm. 2011; 8:6573. [PubMed: 20933098]

105. Grobner T. Gadolinium-a specific trigger for the development of nephrogenic fibrosing dermopathy and nephrogenic systemic fibrosis? Nephrol Dial Transplant. 2006; 21:1104-1108. [PubMed: 16431890]

106. Prince MR, Zhang H, Morris M, et al. Incidence of nephrogenic systemic fibrosis at two large medical centers. Radiology. 2008; 248:807-816. [PubMed: 18710976]

107. Wertman R, Altun E, Martin DR, et al. Risk of nephrogenic systemic fibrosis: evaluation of gadolinium chelate contrast agents at four American universities. Radiology. 2008; 248:799-806. [PubMed: 18632533] 

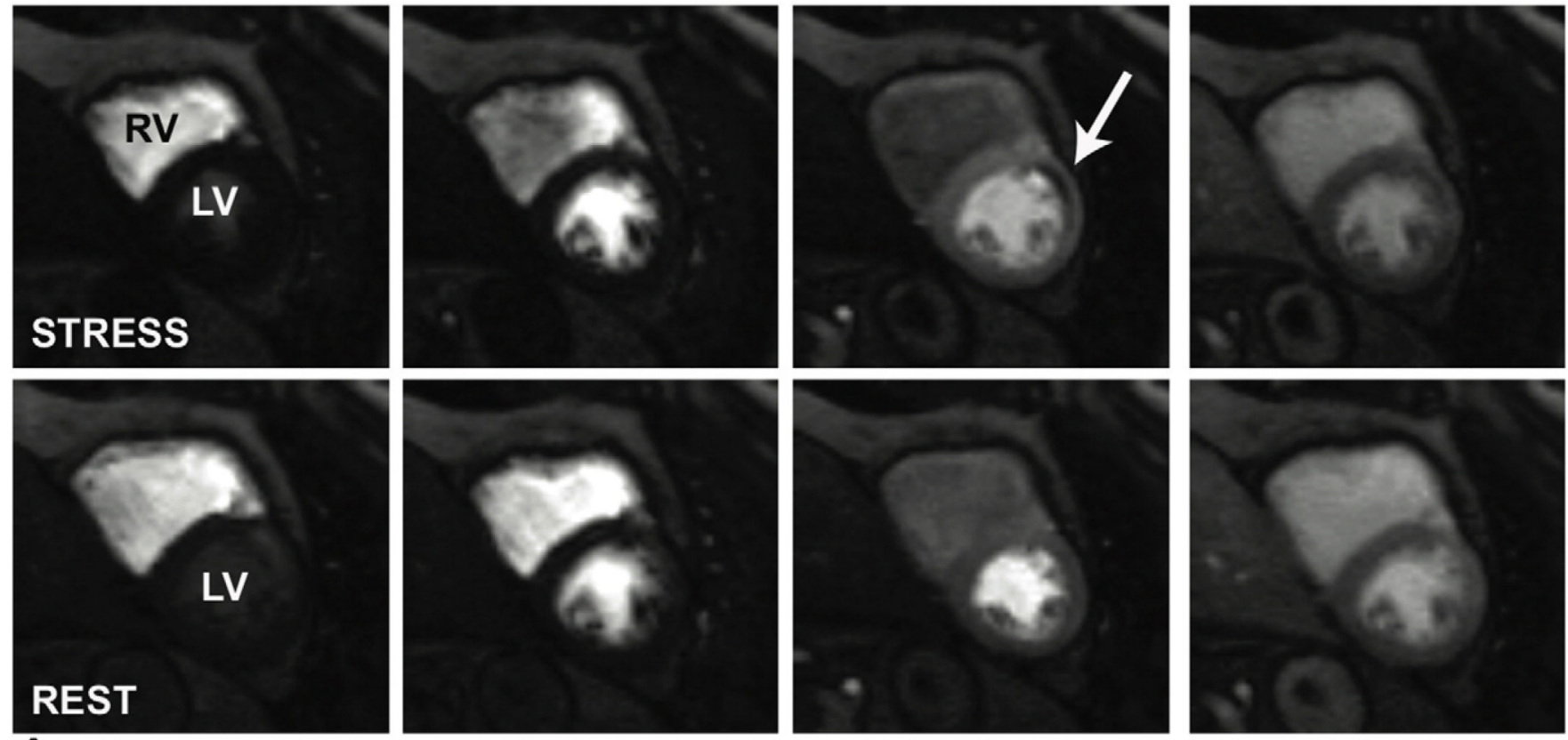

A
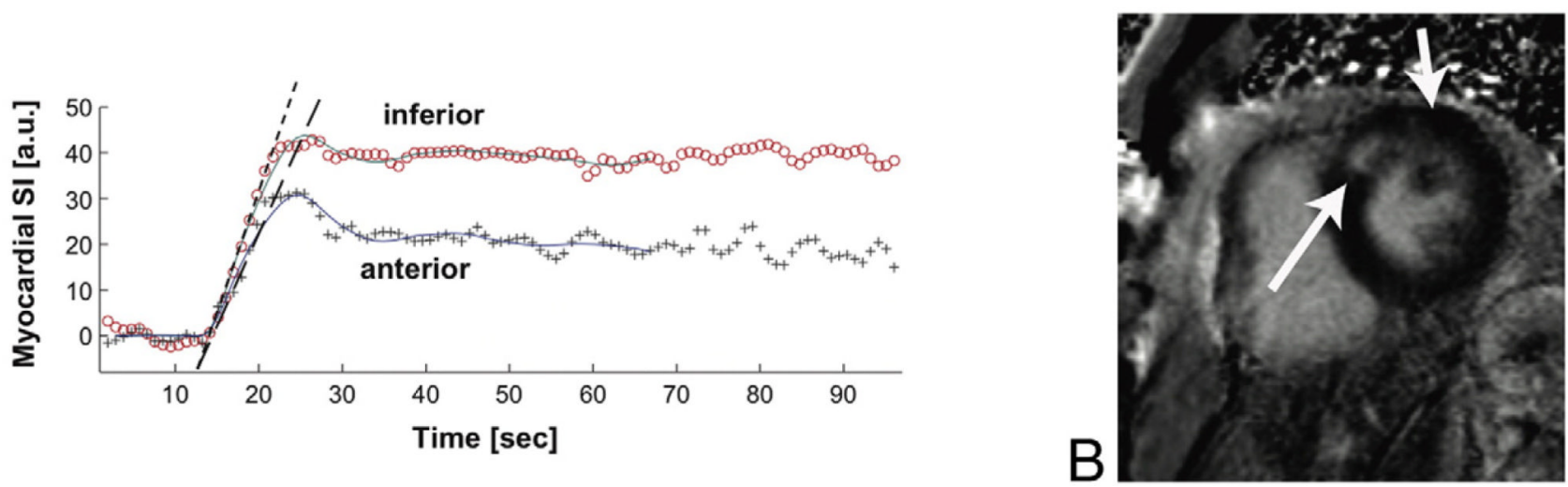

Fig 1.

Demonstration of a myocardial stress perfusion study in a patient who had a prior anteroseptal MI with residual ischemia in the anterior wall. This patient had subsequent cardiac catheterization that demonstrated a significant obstructive lesion in the left anterior descending artery. Panel A demonstrates a midventricular slice of both the left ventricle and right ventricle during stress and rest perfusion imaging. The images progress through time from left to right as gadolinium is being injected during the stress perfusion study. The arrow demonstrates a relative reduction of perfusion in the midanterior wall during the stress phase (top row) but not during the rest phase (bottom row), suggesting midventricular anterior ischemia. Panel B demonstrates LGE in the anteroseptal segment, because of the prior MI, but no LGE in the midanterior segment, suggesting complete viability of this segment. Panel $\mathrm{C}$ demonstrates quantification of the relative signal intensities during the stress myocardial perfusion images between the anterior and inferior segments. The graph shows lower signal intensity within the anterior segment because of the ischemic defect. 


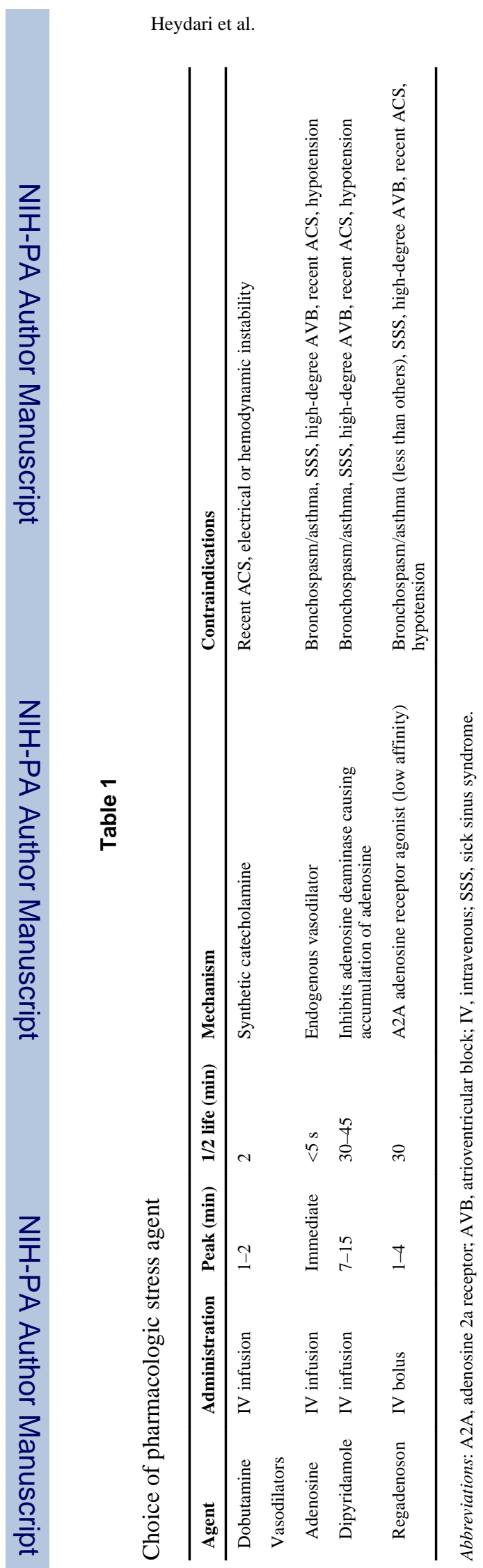

Page 19 


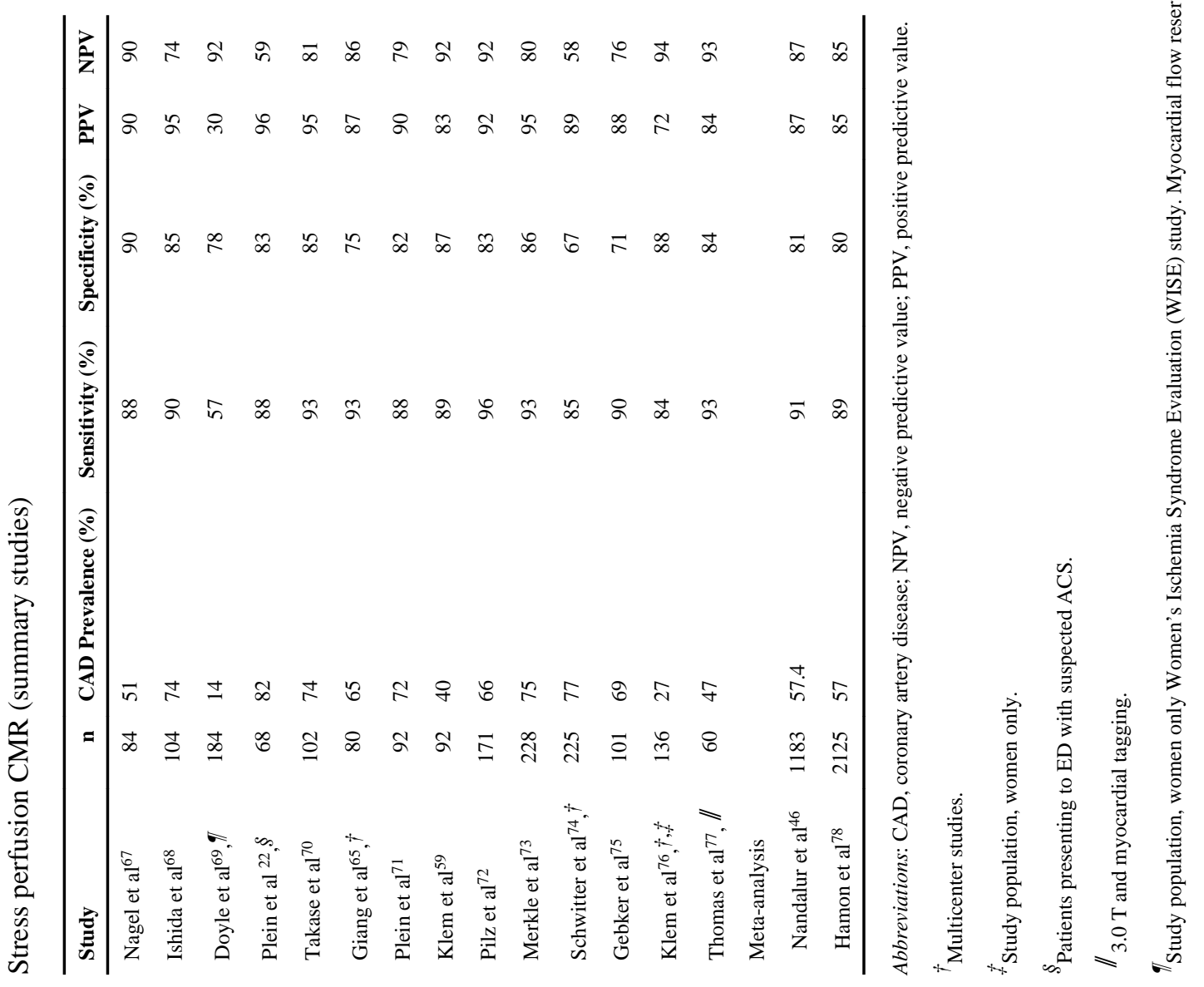

Prog Cardiovasc Dis. Author manuscript; available in PMC 2014 March 14. 